\title{
Princeton Lectures in Analysis by Elias M. Stein and Rami Shakarchi-A Book Review
}

\author{
Reviewed by Charles Fefferman and Robert Fefferman \\ with contributions from Paul Hagelstein, \\ Nataša Pavlović, and Lillian Pierce
}

\section{Comments by Charles Fefferman and Robert Fefferman}

For the last ten years, Eli Stein and Rami Shakarchi have undertaken a labor of love, producing a sequence of intensive undergraduate analysis courses and an accompanying set of four books, called the Princeton Lectures in Analysis. The individual titles are:

- Fourier Analysis: An Introduction

- Complex Analysis

- Real Analysis: Measure Theory, Integration, and Hilbert Spaces

and

- Functional Analysis: Introduction to Further Topics in Analysis.

All four books are now available; all four books bear the unmistakable imprint of Eli Stein.

Every mathematician knows Stein as an analyst of unsurpassed originality and impact. A few dozen of us have had the privilege of writing a Ph.D. thesis under his supervision. We know firsthand how Eli conveys the essential unity of many seemingly disparate ideas. To him, analysis is always an organic whole. Even more remarkably, his own enthusiasm for the subject instills great optimism in all who learn from him. First-rate math is right in front of

Charles Fefferman is the Herbert Jones University Professor of Mathematics at Princeton University. His email address is cf@math.princeton.edu.

Robert Fefferman is the Max Mason Distinguished Service Professor of Mathematics at the University of Chicago. His email address is rfefferm@uchicago.edu.

DOI: http://dx.doi.org/10.1090/noti832 us, ready to be discovered. Eli turns us into research mathematicians by encouraging us to become active participants in the process.

Many who have not enjoyed the privilege of studying under Stein or collaborating with him have nevertheless benefited greatly by studying from his classic books Singular Integrals, Introduction to Fourier Analysis in Euclidean Spaces (with Guido Weiss), and Harmonic Analysis. People far removed from Princeton have been able to read those books and then go on to do significant work. Eli succeeded in putting on the printed page the kernel of what he conveyed as a teacher and research collaborator.

Before proceeding further, we should say a few words about Rami Shakarchi. Shakarchi is a remarkable man in his own right. He is, among other things, a passionate and accomplished pilot. He is now an active worker in the financial sector. As a graduate student, Rami volunteered to help Eli to plan the sequence of courses and to write the four books.

The collaboration was a great success. Eli and Rami got along famously and communicated perfectly. Rami earned his Ph.D. under one of us (CF) and took a demanding finance job in London. Even when his firm, Lehman Brothers, declared bankruptcy, he stayed with the Stein project and saw it through to the end.

The Stein-Shakarchi books constitute an extraordinary achievement. They are accessible (with a lot of work) to any math student who has had a rigorous one-variable calculus course and a little linear algebra, yet they cover an astonishing range of material, including (in alphabetical order) Brownian motion, the Brunn-Minkowski inequality, 
Dirichlet's principle, Dirichlet's theorem on primes in an arithmetic progression, elliptic functions, the ergodic theorem (maximal, mean, and pointwise), the gamma function, the Hardy-Littlewood maximal theorem, Hausdorff dimension, the isoperimetric inequality, the Kakeya problem, the partition function, the prime number theorem, representations of positive integers as sums of two squares and as sums of four squares, the Riemann zeta function, the Runge approximation theorem, Stirling's formula, theta functions, and a lot more.

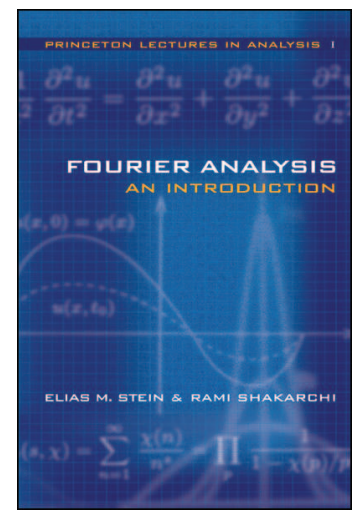

Moreover, these topics appear, not as a zoo of isolated wonders, but quite naturally as part of a unified picture. For instance, results from analytic number theory and probability give the books a relevance that reaches beyond analysis to other branches of mathematics and science.

The material is explained with the perfect clarity, focus on essentials, and stress upon the interconnection of ideas that one expects from Eli Stein. Numerous challenging problems encourage active audience participation. By working the problems, the student earns an understanding of key ideas.

Another remarkable feature of the Stein-Shakarchi books is that they take very seriously the historical development of analysis. The authors boldly present Fourier analysis before passing from the Riemann to the Lebesgue integral. This makes it possible for the student to start doing interesting mathematics right away, without getting bogged down in unmotivated technicalities. When the time comes to start discussing measure and integration, the subject is introduced with the following words:

Starting in about 1870 , a revolutionary change in the conceptual framework of analysis began to take shape, one that ultimately led to a vast transformation and generalization of the understanding of such basic objects as functions, and such notions as continuity, differentiability and integrability.

The earlier view that relevant functions in analysis were given by formulas or other "analytic" expressions, that these functions were by their nature continuous (or nearly so), that by necessity such functions had derivatives at most points, and moreover these were integrable by the accepted methods of integration-all of these ideas began to give way under the weight of various examples and problems that arose in the subject, which could not be ignored and required new concepts to be understood. Parallel with these developments came new insights that were at once more geometric and more abstract: a clearer understanding of the nature of curves, their rectifiability and their extent; also the beginnings of the theory of sets, starting with subsets of the line, the plane, etc., and the "measure" that could be assigned to each.

One sees here the vast and penetrating scope of Stein's view of analysis and how he is able to weave it into his teaching.

The question remains, of course, how such an ambitious set of books can work in practice. To answer this query, we asked Paul Hagelstein, Nataša Pavlović, and Lillian Pierce to comment on their own experiences. Paul taught the Stein-Shakarchi courses as a VIGRE postdoc. Nataša taught Complex Variables à la Stein-Shakarchi at Princeton. Lillian took the Stein-Shakarchi courses as an undergraduate, then went on to serve as a TA for Nataša's class. We are grateful to Paul, Nataša, and Lillian for their thoughtful comments, which appear below.

It remains for us only to add that, in our view, the Stein-Shakarchi books will be immensely valuable for any undergraduate or graduate math student, for a wide audience of working mathematicians, and for many science or engineering students and researchers with a mathematical bent. Even those few mathematicians who thoroughly know the contents of all four books will find pleasure in the beauty of their unified presentation of a vast subject.

\section{Comments by Lillian B. Pierce The Courses}

In the fall of 1999 a murmur spread through the community of math students at Princeton: a new course would be offered in the spring, the first of four intensive courses in analysis, taught by Professor Elias M. Stein. Professor Stein had already acquired status in our eyes, as it happened that most of my cohort had taken his Introduction to Single Variable Real Analysis course in our first semester at Princeton. So as the rumor of the new course rumbled through our ranks, it was clear that we'd all sign up for it. It was also clear from the first lectures that we were in for something unlike anything we'd ever experienced before.

At the beginning of each of the four courses, Professor Stein started by saying that he would work very hard on the course and that the course would in turn require us to work very hard. Then he expressed his gratitude, ahead of time, for all our ensuing hard work. This was a striking attitude of enfranchisement, which I certainly never encountered in any other course.

Indeed, we did have to work hard. Of course, substantial courses in mathematics are often described as "hard", and this superficial descriptor can conceal a multitude of sins or blessings. Are the lectures simply unclear, the assigned problems ill chosen? Or are the lectures deeply insightful, 
the assigned problems a methodology for building a versatile toolbox? In this analysis sequence, the lectures were fluid and painstakingly prepared; they possessed Stein's trademark of logical progressions of big ideas blended with clarifying close-up examinations. In particular, he was careful to lift each concept up from first principles, so that we could start from our existing knowledge (as second-year undergraduates) and follow him deeply into the material. It was as though he was successfully accelerating a car full of passengers, having first checked that all our seatbelts were fastened!

The problem sets were memorably substantial (one group of students estimated that they sometimes spent about thirty hours per week on the courses). But, unlike some "hard" problem sets in ambitious courses, these did not feel punitive. The problems were carefully posed and often with a hint to get us on the right track. The goal of each problem was always some interesting property or beautiful expression that was appealing enough to motivate us to push through to a solution. Along the way, we learned how to use classical analytic techniques. $^{1}$

The courses set a rapid pace, but the lectures did not feel hurried, and they did not sweep details under the rug. Glimpses of the historical development of the field were contained in the presentation of the theory. One day, at the start of class, Professor Stein read aloud some writing of Fourier in the French original and then gave an off-the-cuff translation. I remember feeling at that moment a thrilling connection to the origins of the deep ideas of Fourier analysis. Students are used to their own comprehension of a concept starting out fuzzy, eventually sharpening with thought and practice. But students can also be startled when they first realize that the concepts themselves started out fuzzy and were sharpened by a progression of refinements by a series of mathematicians. The courses conveyed this historical sense, highlighting key contributions

\footnotetext{
${ }^{1}$ The mechanics of the courses contributed to a comfortable, noncompetitive atmosphere. Each week the problem set consisted of a dozen or more required problems, with a few optional problems printed on a separate sheet of colored paper to emphasize their optionality. Hard problems were starred, so we knew not to mind being stymied. Each week we could select one required problem that we were having trouble with and defer handing in the solution until later. We were encouraged to collaborate on problems. These collaborations, which got us "talking math" and learning how to engage in the experimental back-andforth of creative mathematics, led to some of my best times at college. Our solutions were not graded against a strict rubric of potential points to be lost, but were instead labeled $\alpha, \beta$, or $\gamma$-which encouraged us to be creative in our approaches. The exams were not stressful, but instead made sure that we understood the ideas in the main theorems (such as the role each hypothesis played) and gave us a chance to apply these ideas in a few choice, beautiful problems.
}

of individual mathematicians (while avoiding a digression into blow-by-blow timelines).

Looking back, I realize that we students did not feel that we were merely inputting stale mathematics into our heads; the beautiful presentations in the lectures and the intense work on the problem sets made us feel like we were part of a creative force, (re)discovering mathematics. I am sure we had this sense of creation partly because the courses were being developed into books, but I think that it also came from the respect given to the work we were doing, conveyed by the high standards and high expectations of the courses. We felt like fledgling mathematicians, and this gave us a corresponding pleasure and responsibility, which we tried to live up to. I suspect that few people have ever learned material so naturally and so deeply as we did in these courses.

\section{The Books}

As a student, the first time the four courses were taught I did not of course benefit from the books in their final, published form. But the intensity of the courses imbued the material with an indelible presence, and reading the books feels like a homecoming.

Readers of Stein's more advanced books in analysis (say Harmonic Analysis from 1993) will be familiar with the precision and clarity of his presentations. Mathematical writing can be too detailed, submerging big ideas in muddy, over-notated details. Or it can be cruelly sparse, failing to give the reader necessary hand- and footholds up the cliff of comprehension. Stein's writing generally avoids both of these flaws; the Lectures in Analysis series, aimed at undergraduates, is no exception. The mathematics is deep, and of course reading the books (and solving the problems) requires sharp attention and thought, but the pace from sentence to sentence, equation to equation is so carefully and evenly planned that the learning process is consistently rewarding. As one student said, "It's like reading a novel, but by the end you know some mathematics."

The clarity is heightened because concepts are developed from first principles, initially avoiding advanced language (although not sacrificing rigor). For example, the Fourier transform is derived without explicitly calling upon $L^{p}$ spaces. This allows undergraduates to be drawn directly into the power and beauty of Fourier analysis before proceeding through the technical aspects of $L^{p}$ spaces. The definitions and derivations in the text seem so "right" that it is possible to forget that they could ever be described otherwise. Students who go on to graduate studies in math will likely need to review the 
concepts from a more technical perspective, if only to be able to speak of the concepts with the shortcuts that fancier technical language allows. But, by initially learning the concepts free from obscure technical language, they will view the principles of the derivations almost as second nature. Some math texts are handy learning guides or excellent references, but often one has to translate the symbols on the page into one's own internal language in order to store the concepts as retrievable thoughts. The Princeton Lectures in Analysis is much more readily digested.

As in the courses, the "Exercises and Problems" in the books are integral to the experience of learning. The range in difficulty allows for the possibility of both undergraduate and graduate use. The problems are clearly very carefully designed: some codify definitions, some develop dexterity with key theorems, some prepare for deeper material in analysis, some elaborate on classical examples or counterexamples, some push toward applications in other fields. All of them are posed clearly in a self-contained manner that makes them easy to reference (especially by means of the careful index). A reader who solves all the problems will develop a fluid proficiency in analytic methods as well as an arsenal of useful facts and tools. The more casual reader will not be irritated by the relegation of important developments to unsolved "Exercises for the Reader".

One of the goals of the series is to transcend the barriers that arise when the broad field of analysis is artificially broken down into separate subjects in separate textbooks by separate authors. This is certainly achieved: each volume of the series presents a highly organized linear progression of ideas, while the four volumes as a collection are closely interconnected (without repetition) and well cross-referenced. Somewhat unusually, the authors emphasize the interaction between analysis and number theory. Volume I (Fourier Analysis) culminates in a proof of Dirichlet's theorem on primes in arithmetic progressions; Volume II (Complex Analysis) ends in a proof of the prime number theorem and results on theta functions and representations of integers as a sum of two or four squares; Volume IV (Functional Analysis) includes applications to counting lattice points, into which form many problems in number theory may be converted. Some of these presentations are novel, while others are expositions of known proofs, but all share a sharply analytic rigor.

This explicitly analytic presentation of results in number theory absolutely captured my attention as a student; it ultimately is what led me to do graduate work at the intersection of number theory and analysis. Even if one is not interested in number theory per se, an important lesson of the books is that if one is at home with the tools of analysis, one can work "as an analyst" (say, with a concern for integrability, deltas, epsilons, measurability, applying the

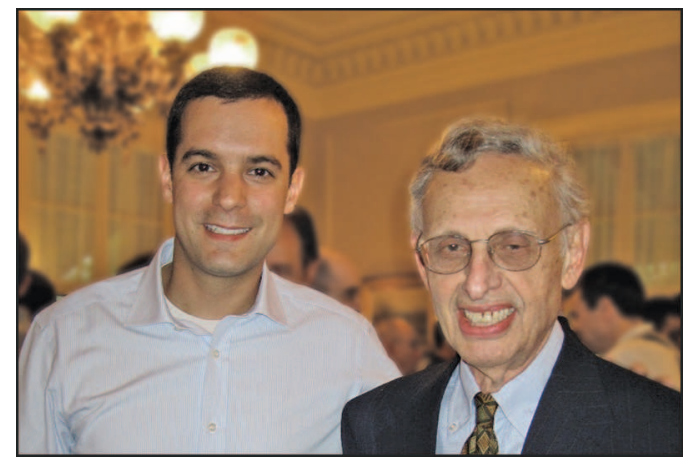

Rami Shakarchi (left) and Elias M. Stein.

tricks of contour integration, oscillatory integrals, etc.), yet prove results originating from a genuinely distinct field (number theory, physics, radiology, and so on). Analysis becomes a mode of working rather than just a set of facts.

While I have used the texts as a student and teaching assistant, I have not yet had the pleasure to teach a full course from them. I look forward to doing so. When proving a theorem, Stein sometimes summons up what he calls the main ingredients of the proof. In these texts, the ingredients of analysis are developed in pure and vivid form, and recipes for combining them into expert dishes are beautifully presented. When recipes are this good, I for one will enjoy cooking from them again and again, probably for the rest of my life.

\section{Comments by Paul Hagelstein}

A fresh Ph.D. from the University of Chicago, I arrived at Princeton University in September 2000 with a three-year VIGRE postdoctoral fellowship. In my letter of appointment, I had been informed that my teaching responsibilities at Princeton would consist of assisting E. M. Stein with the teaching and development of a sequence of undergraduate courses in analysis. As a graduate student I had studied Stein's Fourier Analysis on Euclidean Spaces (coauthored with Guido Weiss), Singular Integrals and Differentiability Properties of Functions, and Harmonic Analysis in great detail and knew them to be masterpieces, the worthy sequels of Zygmund's Trigonometric Series. I knew Stein to be a towering figure in analysis, a man who had done seminal work in numerous important areas: harmonic analysis, partial differential equations, representation theory, and several complex variables, for example. That being said, my advisor, Robert Fefferman, had told me that Stein was working on a new series of books that would be very different from the ones he had written in the past; they would be a series of texts addressing fundamentals of analysis from a highly mature perspective, somewhat akin to what Feynman had done in his Lectures on Physics. I was certainly very excited at having the opportunity 
to participate in this undertaking, which promised to be a unique experience. Immediately after my arrival at Princeton, I introduced myself to Stein, and he enthusiastically welcomed me to the project. Friendly, receptive, and perfectly willing to answer my questions regarding the courses he was teaching, as well as my personal research, he demonstrated immediately that his reputation for graciousness and generosity is well deserved.

On my second day at Princeton, Professor Stein introduced me to Rami Shakarchi, and they filled me in on what was happening with the project. They were collaboratively working on what was to be a series of four books on analysis. The first book was almost completed and was on Fourier analysis. The second book was to be devoted to complex analysis, the third to measure theory and Hilbert spaces, and the last to functional analysis and further topics. The first undergraduate course involving this sequence had been taught the prior spring semester. In the fall of 2000, while Stein was teaching complex analysis, he and Rami would work on the complex analysis text. The sequence of courses would be continued and finished during the 2001-2002 academic year. In the spring of 2001, I was to teach the sequence myself starting with the course in Fourier analysis. In doing so, I would use upper-level drafts of the texts and provide yet another thorough proofreading before publication.

That fall I sat in on all of Professor Stein's lectures in complex analysis and held office hours to answer student questions regarding the course. The pace of play was fast. Stein prepared transparencies outlining the main definitions and theorems of each lecture, initially using the chalkboard to write down the associated proofs. When we realized that the students were having difficulty writing everything down, we decided that copies of the transparencies should be made available to them before each lecture.

The homework sets, divided into so-called Exercises and Problems, were fascinating, a definite strongpoint of this course and indeed of the entire sequence. The Exercises typically involved basic and important calculations (such as the computation of contour integrals) and also introduced students to fundamental mathematical objects and results such as Abel's theorem, Blaschke products, and the Mellin transform. There were some unexpected jewels as well-mathematical and pedagogical surprises that are pleasurably illuminating. The Problems were typically much more involved, sometimes outlining alternative proofs of results in the texts (such as Koebe's proof of the Riemann mapping theorem) and frequently requiring students to get their hands dirty in order to find a solution. I found then, and I continue to find, that even the best students struggle with a number of these, but in the process they are not only learning how to partition problems into more tractable pieces but also getting a better feel for handling chains of inequalities. Of course, Stein and Shakarchi designed these problems well aware that our students are going to need these skills if they wish to be successful researchers in analysis. The Princeton students who were involved in this project were outstanding and exceedingly hardworking. Several of them now have Ph.D.s in mathematics and are already making notable contributions in research. Certainly the excitement and enthusiasm they felt for these courses was much appreciated by Professor Stein, Rami, and me.

According to the original plan, I started independently teaching the sequence of courses in the spring of 2001. Drafts of the texts were few suggestions regarding the texts and are available at this point, and the students and I marked our copies with corrections that were later forwarded to Eli and Rami. I had a particularly exceptional group of six students for the first two courses in the sequence. They made quite a responsible for many of the hints that are now included with the "Exercises and Problems".

A few words about Lectures in

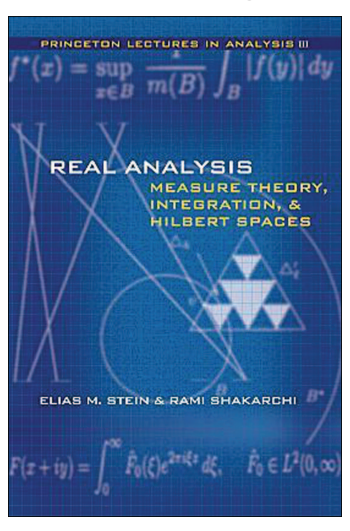
Analysis as a whole. Zygmund, after emphasizing in his Trigonometric Series the impact of harmonic analysis on other areas of mathematics, once joked about his "mathematical imperialism". This theme of imperialism ${ }^{2}$ is clearly evident in the Lectures in Analysis: in the volume Fourier Analysis, Stein and Shakarchi establish the fundamentals and central questions of Fourier analysis as a base camp for many of their further operations. Young analysts should take heed and thoroughly assimilate how basic problems involving heat flow and vibrating strings motivate the notion of a Fourier series and how making precise this notion necessitates the introduction of families of good kernels and the concepts of pointwise and norm convergence. Having done so, they will be in a much better position to appreciate subsequent, more abstract, results regarding the convergence of sequences and series and the Lebesgue theory of integration. They will also be able to validate these results against personal experience gained in the study of elementary Fourier series.

Having established this base camp in Fourier Analysis, the authors take advantage of it repeatedly in later volumes. In Complex Analysis, they show how the Fourier transform provides a condition that guarantees a function $f$ defined on the real line can be extended to a holomorphic function in the complex plane. In Real Analysis they show that the heat and Fejér kernels provide approximations

\footnotetext{
${ }^{2}$ For Zygmund's views on the matter, see the epigraph in the chapter on distributions in Functional Analysis.
} 
to the identity; they relate orthogonality considerations in Fourier series to Hilbert spaces and use the Radon transform to show why Besicovitch sets in $\mathbb{R}^{2}$ have dimension two. In Functional Analysis, issues involving the pointwise divergence of Fourier series illustrate the uniform boundedness principle, and the desire to extend the Fourier $L^{2}$ theory to $L^{p}$ spaces motivates the Riesz interpolation theorem, which in turn is used to develop the $L^{p}$ theory of the Hilbert transform. In the same volume, Stein and Shakarchi also consider issues involving the restrictions of Fourier transforms and how they relate to dispersive partial differential equations.

Finally, the incredibly broad mathematics for which Stein is so well known is beautifully reflected in this series. A surprising amount of number theory

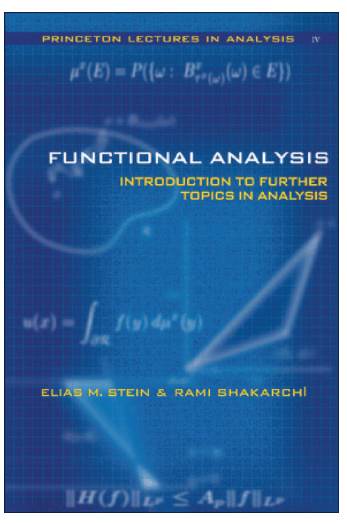

is treated, including Dirichlet's theorem on primes in arithmetic progressions, the two and four square theorems, the prime number theorem, and results related to counting lattice points (along the lines of the Dirichlet divisor problem). Not only are the basics of complex analysis and measure theory covered, but the series also introduces students to fundamentals of ergodic theory, spectral theory, probability theory, Brownian motion, and several complex variables. One particularly nice aspect of the topics treated is that students who reach the end of the last volume have made significant headway toward the research frontier in a number of areas, including those involving Bochner-Riesz summability, the dimension of Besicovitch sets in $\mathbb{R}^{n}$, regularity properties for X-ray like transforms, and lattice point counting. These research areas have become quite central, and even seasoned analysts who wish to learn about these topics will find much of interest here.

Who is the target audience for these books? The four volumes of Stein and Shakarchi require surprisingly little in prerequisites-essentially a solid background in calculus with a rudimentary exposure to linear algebra. I recommend that aspiring mathematicians get copies into their hands as soon as possible. The books are clearly written and are eminently suitable for self-study. Moreover, many of the important topics covered in these volumes are only intermittently taught in even the best graduate programs. Stein and Shakarchi have served young mathematicians well in providing an introduction to these topics in such a cohesive manner.

Functional Analysis will likely become standard reading for graduate students in harmonic analysis. In many respects it provides a useful companion volume to Stein's Singular Integrals and Harmonic Analysis. Like these earlier volumes but at a more leisurely pace, Functional Analysis treats the Hilbert transform, the Riesz interpolation theorem, Hardy spaces, BMO, and oscillatory integrals and their applications to dispersive equations. Moreover, since there are profound connections between harmonic analysis and the all-too-often unfamiliar realm of several complex variables, students of the former will greatly appreciate the chapter on the latter; it not only introduces the subject but also clearly indicates where to go to learn it further.

Analysis is a beautiful and extremely wideranging subject, extending from the oscillations of vibrating strings to number theory and from the splashing of waves to the pricing of stock options. A gestalt overview is difficult to come by; the fabled seven years is an understatement. After decades of study and research, E. M. Stein is one of the very few who have attained such a wide-ranging perspective. We are extremely fortunate that he and Shakarchi share it with us in these Lectures in Analysis.

\section{Comments by Nataša Pavlović}

I truly enjoy teaching complex analysis. The power and elegance of its theorems have never ceased to fascinate me (even after I learned that some of them fit into the broader context of the theory of elliptic PDEs). I like to share this excitement with my students. Stein and Shakarchi's book Complex Analysis helps me to do so. From the fact that a holomorphic function is infinitely differentiable to the phenomenon of analytic continuation, complex analysis is (especially for the student accustomed to real variables) rife with magic. And that is how Stein and Shakarchi see the results of complex analysis. In the introduction the authors say: "When we begin the study of complex analysis we enter a marvelous world, full of wonderful insights. We are tempted to use the adjective 'magical', or even 'miraculous', when describing the first theorems we learn; and, in pursuing the subject, we continue to be astonished by the elegance and sweep of the results."

Stein and Shakarchi lead the reader through this magical world of fundamental concepts in complex analysis and some of its important applications in Fourier analysis, number theory, and combinatorics in a gentle and thought-inspiring way. For example, they start by introducing functions in the complex plane and the concept of integration along curves, and in the subsequent chapter they link these two notions via Cauchy's theorem. Their approach is gradual and as such it is perfect for the classroomlike a chorus in a Greek play, at the beginning of a chapter they give the reader a quick preview of what is coming as well as a glimpse of applications of the results to be discussed. Then the story develops from simpler building blocks to more involved results. For example, in the above-mentioned chapter on Cauchy's theorem, the authors first familiarize the reader with the special case of Cauchy's theorem for a triangle and then use this as a starting point 
to derive many other results. The chapter proceeds with a detailed evaluation of some integrals (among them is a classical example that shows that $e^{-\pi x^{2}}$ is its own Fourier transform) and concludes with applications, beginning with the fact that a holomorphic function in an open set $\Omega$ has a power series expansion for all points in a disc whose closure is contained in $\Omega$ and proceeding to Liouville's theorem and an elegant proof of the fundamental theorem of algebra based upon it. A gradual approach applies to all subsequent chapters as well as to the entire book. Proofs throughout are elegant and clearly written-it is a joy to follow them.

The book differs significantly from many other undergraduate or beginning graduate textbooks, because Stein and Shakarchi continually remind the reader of links with previously discussed topics and offer a perspective as to why these results are relevant for further applications. Hence a student can learn about motivations and connections in greater detail than he/she generally does in lectures.

Another difference with many other complex analysis textbooks, which makes this one almost a page-turner, is the choice of more advanced topics. For example, one chapter is devoted to establishing a connection between the 1D theory of the Fourier transform and complex analysis, while another chapter focuses on the zeta function and a proof of the prime number theorem. When I used the book at Princeton University to teach an advanced undergraduate class, students really appreciated these connections with Fourier analysis and number theory.

At Princeton, the Complex Analysis course consisted of two components: lectures and weekly problem sessions. Lillian Pierce, who did a wonderful job leading the problem sessions affiliated with my lectures, discussed problems which mainly came from the book itself. She was able to do so because, in addition to thoroughly solved examples in the main text, a nice selection of exercises follows each chapter. Among them are important types of problems which include both easy and more elaborate ones (for which a hint is often given). Also most chapters have a list of more challenging exercises, entitled "Problems".

Many different audiences can benefit from this book. At Princeton the textbook is used for an advanced undergraduate course, while at some other universities it is used as a beginning graduate textbook. With its clear presentation and broad range of exercises, this text is a "must read". I myself recommend it as supplemental reading for students in a beginning undergraduate course in complex analysis. $^{3}$ Since it is less dense and provides a

\footnotetext{
${ }^{3}$ For example, when trying to teach my students that one cannot compare complex numbers, I typically refer to the exercise in the book of Stein and Shakarchi that guides
}

wealth of applications, the book is more accessible than Ahlfors's Complex Analysis or Rudin's Real and Complex Analysis. On the other hand, with its clear presentation of fundamental concepts of complex analysis, as well as their applications to algebra, Fourier analysis, number theory, and combinatorics, and a wealth of important as well as interesting exercises, I like to recommend the book as essential and valuable to mathematics and physics graduate students seeking a classical book on complex analysis. It is such a beautifully written book, with a wonderful balance between insight and technical rigor, that even research mathematicians will be proud to have it on their bookshelves. Those contemplating writing a mathematical textbook might like to have an additional copy on their nightstand too.

\section{Closing Remarks}

The books of Stein and Shakarchi are a remarkable achievement, the crowning glory of a very distinguished and important career in analysis. They pass on to future generations a wealth of collected wisdom and a fusillade of techniques that will help to launch many a productive research career. Our lives are all enriched by these books.

students in showing that it is impossible to define a total ordering in $\mathbb{C}$ compatible with its structure as a field. 